\title{
D-03
}

\section{TRANSFERENCIA DE RESULTADOS DE INVESTIGACIÓN PARA EL AHORRO DE AGUA Y DE LA ENERGIA EN COMUNIDADES DE REGANTES A TRAVÉS DEL ENTORNO DE GESTIÓN INTEGRADA CORENET-COREGEST}

\author{
Ortiz Ruiz del Castillo, José (P), Aliod Sebastián, R.,Mora Gómez, M., Abadía Sánchez, R, \\ Intrigliolo, D.S.
}

${ }^{1}$ Director, Serina, Servicios de Ingeniería y Arquitectura, S.L, San Marcos, 29, Madrid, jortiz@serina.es. ${ }^{2}$ Profesor Titular, Universidad de Zaragoza, Área Mecánica de Fluidos, raliod@unizar.es ${ }^{3}$ Dr. Ingeniero Agrónomo, Moval Agroingeniería S.L. mmora@moval.es ${ }^{4}$ Director Escuela Ingeniería, Universidad Miguel Hernández, abadia@umh.es ${ }^{5}$ Científico titular, CEBAS-CSIC. dintri@cebas.csic.es

\section{Resumen}

En la actualidad se está produciendo un incremento en la demanda para realizar una gestión optimizada del agua y de la energía empleadas en el regadío. Esto ha dado lugar a una importante actividad de I+D con diferentes sistemas, productos y servicios, que comparten el objetivo de mejorar la aplicación del agua y/o de la energía. Sin embargo, el conocimiento generado choca con dificultades a la hora de transferir los resultados hacia los usuarios finales. Esto es debido, al menos en parte, a la necesidad de que las Comunidades de Regantes cambien su modelo de gestión y sean capaces de integrar los resultados de este conocimiento con los procesos normales de gestión que se emplean en las mismas.

SERINA ha desarrollado la metodología y el Enterprsise Resource Planning (ERP) de gestión CORENET-COREGEST, que permite modernizar los procesos de gestión de las Comunidades de Regantes e integrar herramientas/sistemas/servicios externos generados por los centros de I+D con el objetivo de mejorar la gestión, fundamentalmente, del agua y de la energía.

En este trabajo se muestran algunos casos que ilustran lo anterior y se explican las ventajas para las Comunidades de Regantes usuarias así como por los centros de I+D que han integrado sus productos en CORENET-COREGEST.

\section{Introducción}

En el contexto actual existe una importante presión para que el regadío sea cada vez más eficaz en su uso de agua. Por otro lado, la situación tarifaria de la energía eléctrica obliga a una minimización en el uso de este recurso. También se exige la minimización del impacto medioambiental debido a la aplicación en campo de productos agroquímicos y a otros factores.

Por otro lado, las actividades de modernización de infraestructuras están convirtiendo a las Comunidades de Regantes en entidades cuya gestión tiene que ser más profesionalizada que antaño debido a la introducción de costosas infraestructuras y tecnologías sofisticadas. Todo esto obliga a que las Comunidades de Regantes comiencen a preocuparse por mejorar sus procedimientos de gestión para adaptarse a estos nuevos desafíos. 
Una Comunidad de Regantes tiene que atender, coordinadamente, bajo el punto de vista de gestión los siguientes aspectos: Personas (Regantes, Administraciones, trabajadores), Agua, Energía, Territorio, Tecnología, Infraestructura, Grandes Presupuestos, Documentación.

Tradicionalmente, estos aspectos se han gestionado de forma estanca e individual, sin tener en cuenta que las decisiones que se toman en relación a cada uno de ellos repercute en los otros de forma directa (por ejemplo: el vínculo entre consumo de agua y energía o las posibilidades de disminución en el consumo de agua que se producen cuando se aplica una dosificación optimizada a los cultivos para lo que es necesario conocer la estructura de cultivos). Este enfoque de gestión individual no aprovecha la sinergia combinada que se produce cuando se aborda la gestión de una Comunidad de Regantes de forma global o integral gracias a la cual los gestores pueden tener una visión general del funcionamiento de la Comunidad y optimizar su rendimiento adaptándolo a la nueva situación en la cual se desenvuelven.

Por tanto, es necesario cambiar el estilo de gestión hacia una gestión integral de todos los aspectos que hay que administrar en la Comunidad de Regantes. Esto se traduce en una disminución de los costes anuales para el regante, en un mejor servicio al mismo, en una utilización más controlada de los recursos agua y energía y en una reducción del impacto ambiental del regadío.

La implantación de una filosofía de gestión integral en una Comunidad de Regantes requiere:

- El diseño e implantación de procesos de gestión capaces de responder a las necesidades de la Comunidad de una forma integrada

- La implantación de herramientas de gestión integrada basadas en tecnologías de la información y comunicación tipo ERP que den soporte, formalicen los procesos de gestión y permitan una explotación de los datos correspondientes a distintos procesos de gestión para obtener información sofisticada mediante la combinación de los mismos, capaz de apoyar la toma de decisiones. Estas herramientas deben ser capaces de comunicarse e integrar información de otras herramientas y sistemas externos con el objetivo de facilitar y optimizar los resultados de gestión.

Las herramientas CORENET-COREGEST constituyen un sistema abierto, que cumple todos los requisitos anteriores y que es capaz de comunicarse con herramientas externas e integrar los resultados de I+D que provengan de Centros de Investigación y Universidades que puedan suponer una mejora en la gestión de cualquiera de los procesos de negocio de una Comunidad de Regantes.

CORENET-COREGEST está ya implantado en un elevado número de Comunidades de Regantes y éste número sigue creciendo a buen ritmo por lo que constituye una eficaz manera de transmitir estos resultados de I+D a los usuarios finales de los mismos.

A continuación se describen algunos ejemplos en los que el conocimiento, herramientas y servicios de centros de I+D y universidades ha sido incorporado a CORENET-COREGEST para su uso por Comunidades de Regantes y Regantes. 


\section{Caso 1: Módulo de cálculo de indicadores para gestión de agua y energía. Colaboración CORENET-COREGEST y Universidad Miguel Hernández}

CORENET-COREGEST dispone de herramientas software que permiten la generación de indicadores para la gestión eficiente del agua y de la energía en las que se emplea el conocimiento desarrollado por el Departamento de Ingeniería de la Universidad Miguel Hernández (Escuela Politécnica Superior de Orihuela). Este módulo es utilizable por cualquier Comunidad usuaria de CORENET-COREGEST siendo tanto más indicado su uso cuanto más elevado sea el consumo energético de la Comunidad.

Mediante este módulo los gestores de una Comunidad de Regantes pueden disponer de información tanto de la campaña corriente como información histórica que les permita:

- Realizar un seguimiento de los costes de sus contratos eléctricos

- Disponer de información sobre el funcionamiento de los bombeos para proceder a su reparación preventiva en caso de consumo excesivo.

- Evaluar la eficiencia general de la red de riego en cuanto a distribución de agua, detectando pérdidas.

- Disponer de información sobre el agua teórica que necesitarían los cultivos y compararla con el agua real suministrada.

Este módulo genera indicadores de los siguientes tipos para poder cumplir su objetivo:

- Indicadores de rendimiento de uso del agua:

- De la eficiencia de distribución del agua

- Del consumo de agua de los cultivos

- De adecuación del suministro de agua a las necesidades de los cultivos

- Indicadores energéticos:

- De tipo individual por cada contrato eléctrico (mensuales y anuales)

o De tipo individual por cada estación de bombeo (de seguimiento mensual y de sus parámetros de funcionamiento instantáneo)

o De tipo general para toda la Comunidad de Regantes.

La figura siguiente muestra una pantalla de CORENET-COREGEST correspondiente al módulo de cálculo de indicadores de agua y energía.
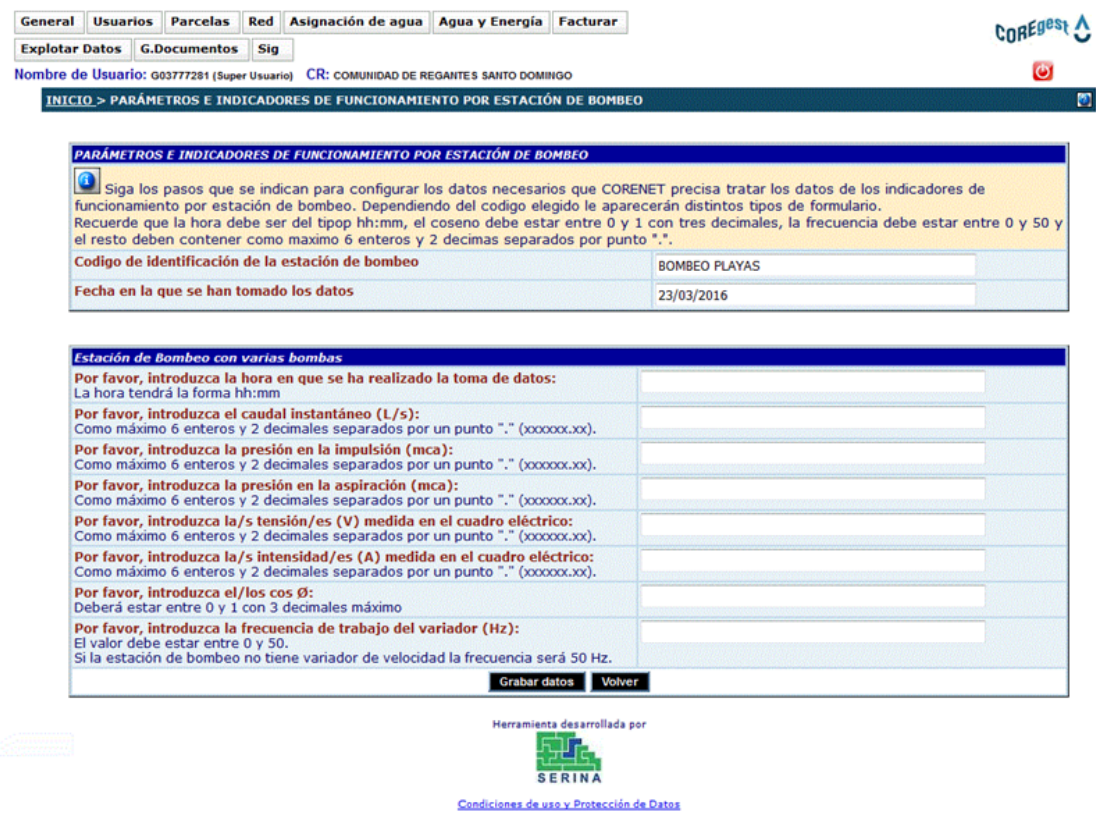
El funcionamiento del módulo requiere datos de entrada que se utilizan de modo exclusivo para el funcionamiento del mismo, siendo necesario establecer en la Comunidad procedimientos de gestión que los recojan. Algunos de estos datos son:

- Extensión temporal de la Campaña de Riego

- Datos sobre los contratos eléctricos existentes en la Comunidad

- Información sobre las estaciones de bombeo

- Coeficientes de cultivo $(\mathrm{Kc})$ para los cultivos que se han recogido previamente en la declaración de cultivos de la Comunidad

- Información de facturas eléctricas

- Volumen de agua acumulado en las balsas

- Volumen de agua acumulado por tomas

- Datos agroclimáticos, evapotranspiración de referencia (ETo) y precipitación media durante la campaña

- Diversos parámetros de seguimiento de los bombeos

En el caso en que exista un SCADA en la Comunidad que recoja todos o algunos de estos datos, resulta posible que el módulo de agua-energía tome la información directamente desde el mismo, simplificando el proceso de recopilación e introducción manual de la información necesaria. Es conveniente considerar este aspecto de manera previa a la instalación del SCADA.

Adicionalmente el módulo necesita información no exclusiva y que se genera en otros procesos de gestión habituales y básicos de la Comunidad tales como la asignación de cupos de riego, declaración de cultivos, gestión de diferentes tipos de agua, etc. CORENETCOREGEST se encarga de transferir al módulo ésta información. Algunos ejemplos de la misma son los siguientes:

- Modelo de la red de riego para trazabilidad de agua.

- Parcelario actualizado con cultivos y superficies ocupadas por los mismos.

- Agua suministrada a cada cultivo por la Comunidad.

Por tanto, a la vista de lo anterior, hay que resaltar que el módulo de agua-energía se ha desarrollado en base a los resultados de investigación que lo han generado, habiéndose implementado dentro de un entorno de gestión CORENET-COREGEST, lo que permite normalizar su utilización dentro de los procesos habituales de gestión de la Comunidad de Regantes, evitando la introducción duplicada de datos para su funcionamiento.

\section{Caso 2: Predicción de necesidades de riego en la Comunidad de Regantes del Canal de Orellana. Colaboración CORENET-COREGEST y CEBAS-CSICIIVIA}

La Comunidad de Regantes del Canal de Orellana es una Comunidad muy extensa (42.000 ha) que se riega a partir del Canal de Orellana mediante una red de acequias recientemente modernizada por la SEIASA.

Como parte de las obras de modernización se ha realizado un inventario de la red de Riego así como la determinación del parcelario empleando referencias catastrales de la Comunidad. Este trabajo ha sido realizado por TRAGSA, y sus resultados han sido cargados en un SIG así como en CORENET-COREGEST. 
Para la gestión integral de la Comunidad se ha implantado CORENET-COREGEST para cubrir las siguientes áreas:

- Gestión Económica Administrativa

- Gestión y control de personal

- Gestión y control del agua

- Gestión de costes de mantenimiento.

Para posibilitar el control y la gestión del agua se han llevado a cabo las actuaciones siguientes:

- Implantación de elementos de medida consistentes en compuertas monitorizadas y caudalímetros en puntos críticos de la red que vuelcan sus datos de medida en un servidor situado en la Comunidad. Estos datos se incorporan también a CORENETCOREGEST a partir de un módulo software específicamente desarrollado al efecto. De esta manera, a través de la modelización de la red de riego existente en CORENTE-COREGEST es posible disponer de informes de uso del agua suministrada por las distintas acequias.

- Implantación de una metodología de determinación empírica de las necesidades de riego, basada en resultados de I+D del IVIA (Instituto Valenciano de Investigaciones Agrarias) y del CEBAS-CSIC en colaboración con el Centro de Investigaciones Científicas y Tecnológicas de Extremadura (CICyTEX). Esta metodología ha sido integrada en CORENET-COREGEST y para su funcionamiento utiliza datos que se recogen anualmente durante el proceso de declaración de cultivos y carga en CORENET-COREGEST el modelo de la red de riego para una trazabilidad del agua integrada en CORENET-COREGEST. Esta metodología empírica permite:

- Predecir las necesidades de riego en cada parcela y para cada cultivo en función de parámetros tales como la variedad del cultivo, el tipo de suelo, tipo de riego empleado, precipitación efectiva, etc.

- Predecir para cada acequia las necesidades de suministro de agua en función de los cultivos que se riegan y sus características.

Gracias a esta información es posible:

- Comparar el consumo de agua por acequias con el consumo teórico comprobando así si se está realizando una gestión adecuada del agua de riego.

- Sensibilizar al regante a través de la oficina electrónica del regante de CORENETCOREGEST para un uso del agua más racional.

- Permitir la programación de riegos en parcela basándose en criterios agronómicos de necesidades de los cultivos.

- Predecir las necesidades de riego de la Comunidad para apoyar las solicitudes de agua al Canal de Orellana, evitando las posibles peticiones excesivas.

La metodología empleada para el cálculo de las necesidades de riego ha sido la propuesta por la Organización de las Naciones Unidas para la Alimentación y la Agricultura (FAO), recogida en la publicación de la serie Riego y Drenaje 56; Evapotranspiración del cultivo: Guías para la determinación de los requerimientos de agua de los cultivos. (Allen et al. 1998), estimando las necesidades hídricas mediante un procedimiento que tiene en cuenta dos factores: 
1) Variables climáticas (temperatura y humedad del aire, radiación solar y velocidad del viento) que influyen en la demanda evaporativa o evapotranspiración de referencia (ETo)

2) Un factor ligado al cultivo, denominado coeficiente del cultivo (Kc).

Bajo este modelo, las necesidades hídricas o evapotranspiración del cultivo (ETc) se calculan como ETc $=\mathrm{ETo} * \mathrm{Kc}$.

Los coeficientes de cultivo empleados para obtener las necesidades hídricas de los cultivos se resumen en los puntos siguientes:

- Coeficientes de cultivo (leñosos y hortícolas) expresados en función de los meses de año. Propuestos por Villalobos et al. en el "Estudio Sobre Necesidades de Agua de Riego de los Cultivos en la Zona del Transvase Tajo - Segura".

- Coeficientes de cultivo (leñosos, hortícolas y otros) divididos en tres etapas $\left(K_{\mathrm{c}}\right.$ inicial, $K_{\mathrm{c}}$ medio y $K_{\mathrm{c}}$ final), propuestos por Allen et al. (1998). Crop evapotranspiration. Guidelines for computing crop water requirements. Irrigation and Drainage Paper 48. FAO, Roma, Italia. En esta hoja también se presenta la altura y profundidad máxima de los cultivos, la fracción de agotamiento de agua permisible en el suelo, el umbral de salinidad máxima a partir del cual la producción se ve negativamente afectada y las necesidades de lavado.

- Coeficientes de cultivo (leñosos y hortícolas) expresados en función del periodo fenológico del cultivo. Obtenidos del Servicio de Información Agraria de la Región de Murcia (SIAM). La dirección web de acceso es http://siam.imida.es/apex/f?p=101:39:8087656247004068.

- Coeficientes de cultivo (leñosos, hortícolas y otros) expresados en función de los meses de año. Obtenidos del sistema de información agroclimática para el regadío (SIAR). La dirección web de acceso es http://www.magrama.gob.es/es/agua/temas/observatorio-del-regadio-

espanol/sistema-de-informacion-agroclimatica-para-el-regadio/.

- Coeficientes de cultivo leñosos expresados en función de los meses de año. Propuestos por el Instituto Valenciano de Investigaciones Agrarias (IVIA). La dirección web de acceso es http://riegos.ivia.es

- Coeficientes de cultivo hortícolas expresados en función de las semanas de cultivo. Propuestos por el Instituto Valenciano de Investigaciones Agrarias (IVIA). La dirección web de acceso es http://riegos.ivia.es

Hay que resaltar que para el cálculo de las dosificaciones óptimas, ha sido necesaria la utilización de los resultados de investigación del CEBAS/IVIA, integrándolos dentro del entorno CORENET-COREGEST. Para ello ha sido necesario modificar el procedimiento de declaración de cultivos para recopilar la información necesaria para el cálculo. Esta modificación sólo le supone un pequeño trabajo adicional a la Comunidad que se ve compensado por la información relativa al uso del agua que se genera.

\section{Caso 3: Optimización de turnos de riego bajo el punto de vista energético en la Comunidad de Regantes del Pantano Estrecho de Peñarroya. Colaboración CORENET-COREGEST y Universidad de Zaragoza.}

La Comunidad de Regantes del Pantano Estrecho de Peñarroya se extiende sobre $7.000 \mathrm{Ha}$ y riega mediante redes a presión con un gran consumo energético. Toma el agua del 
Pantano Estrecho de Peñarroya. Los usuarios riegan por turnos que se venían organizando por el propio personal de la Comunidad en función de la capacidad de la red.La Comunidad dispone de cinco sectores con riego automatizado de forma independiente en cada sector empleando sistemas de Motorola.

La Comunidad emplea CORENET-COREGEST para su gestión integral, habiéndose empleado un proceso de control de asignación del agua a los usuarios mediante la herramienta de gestión de cupos de agua de CORENET-COREGEST que limita el agua que puede suministrarse a cada usuario en función de la superficie de sus parcelas y otros parámetros.

Debido al elevado gasto económico que supone el consumo de energía, se tomó la decisión de establecer un mecanismo de concesión de los turnos de riego que no solamente tuviese en cuenta la capacidad de suministro de la red sino también la minimización del coste de la energía.

Para esto, se ha contado con la herramienta TELEGESTAR, desarrollada por la Universidad de Zaragoza (Aliod et al. 2015) cuyo objetivo consiste en realizar, a partir de las peticiones de riego registradas, una reordenación de las mismas que garantice un coste económico minimizado compatible con la capacidad de la red de riego.

La implantación de TELEGESTAR y los procedimientos de gestión asociados se han realizado con la colaboración de la empresa MOVAL Agroingeniería, especializada en la implantación de CORENET-COREGEST.

La implantación de TELEGESTAR ha requerido que la funcionalidad de esta herramienta se compatibilizase con CORENET-COREGEST de la forma siguiente:

- En CORENET-COREGEST se recogen las peticiones de riego realizadas por los regantes. Para poder tramitar una petición de riego, CORENET-COREGEST comprueba que el regante disponga de cupo de agua y no esté en situación de morosidad.

- Semanalmente se envía un archivo a los servidores de TELEGESTAR que contiene la información de peticiones de riego así como otra información adicional requerida por TELEGESTAR, tal como características de los contratos eléctricos, etc.

- TELEGESTAR procesa esta información y devuelve las peticiones de riego reordenados a COREGEST. Estas programaciones se incorporan a una herramienta que permite su edición manual por parte del personal de gestión de la Comunidad.

- Una vez se aprueba la reordenación, ésta se incorpora ya a COREGEST, detrayendo las peticiones de los cupos de agua y se envía a los SCADA para la apertura de las válvulas correspondientes.

\section{Conclusiones}

La integración de metodologías y herramientas desarrolladas por centros de I+D en los procesos de gestión de Comunidades de Regantes usuarias de CORENET-COREGEST, constituye una forma viable y práctica de transferir los resultados de investigación al usuario final (Comunidad de Regantes), así como lograr una mayor sostenibilidad del regadío a través de una disminución de costes asociados al uso del agua y la energía. Igualmente, permite la evaluación de estos resultados de investigación a través de la experiencia que se obtiene con su utilización en casos reales. 


\section{Bibliografía}

Aliod R., García S., Faci E., Paño J., Seral P., García A. (2015) "Algorithms and tools for optimum scheduling of on-demand irrigation for an effective energy cost reduction" ICID2015 Conference, Montpellier

Allen, R.G., Pereira, L.S., Raes, D., Smith, M., 1998. Crop evapotranspiration. Guidelines for computing crop water requirements. Irrigation and Drainage Paper 56, FAO, Rome, pp. 300.Shuttleworth, W.J. and Wallace, J.S., 2009. Calculating the water requirements of irrigated crops in Australia using the Matt-Shuttleworth approach. Transactions of the American Society of Agricultural and Biological Engineers 52 (6), 1895-1906.

Allen et al. (1998). Crop evapotranspiration. Guidelines for computing crop water requirements. Irrigation and Drainage Paper 48. FAO, Roma, Italia..

Francisco J. Villalobos, Francisco Orgaz y Elías Fereres. "Estudio Sobre Necesidades de Agua de Riego de los Cultivos en la Zona del Transvase Tajo - Segura"

Coeficientes de cultivo (leñosos y hortícolas) expresados en función del periodo fenológico del cultivo. Obtenidos del Servicio de Información Agraria de la Región de Murcia (SIAM). La dirección web de acceso es http://siam.imida.es/apex/f?p=101:39:8087656247004068.

Coeficientes de cultivo (leñosos, hortícolas y otros) expresados en función de los meses de año. Obtenidos del sistema de información agroclimática para el regadío (SIAR). La dirección web de acceso es http://www.magrama.gob.es/es/agua/temas/observatorio-delregadio-espanol/sistema-de-informacion-agroclimatica-para-el-regadio/.

Coeficientes de cultivo leñosos expresados en función de los meses de año. Propuestos por el Instituto Valenciano de Investigaciones Agrarias (IVIA). La dirección web de acceso es http://riegos.ivia.es

Coeficientes de cultivo hortícolas expresados en función de las semanas de cultivo. Propuestos por el Instituto Valenciano de Investigaciones Agrarias (IVIA). La dirección web de acceso es http://riegos.ivia.es 